\title{
THE PROSPECT FOR NON-SURGICAL DRAINAGE PROCEDURES
}

There has been a surge of interest in alternative means of performing sclerostomies by non-surgical methods in the last 5 years. It has led some ophthalmologists to believe that lasers will replace conventional surgery in the next decade. The idea was made attractive by promises of a less invasive operation with in particular a minimum of conjunctival tissue manipulation, a faster outpatient procedure and an ease of repeatability in previously treated eyes. As the adoption of such techniques would require an initial capital expenditure for the laser apparatus, a potential market has been identified by the commercial sector, which has begun intensive marketing and publicity on a worldwide basis. Health costs are therefore a consideration in the decision to adopt these procedures. There is an increasing interest in this field, and this issue of Eye contains a paper by Allan on the use of excimer laser sclerostomies.

To a certain extent most of the laser sclerostomy techniques currently presenting themselves as candidates for widespread acceptance claim to possess the advantages listed above. However, the main criteria by which they will be judged will be their success rates in performing fistulising surgery compared with the gold standard of the results of surgical trabeculectomy. The other factor critical to their proliferation is the adaptability of these alternative methods of fistula creation to treating diverse forms of glaucoma successfully.

Allan graded the various methods by their ability to cut sclerostomies consistently and accurately yet with the minimum amount of collateral tissue damage from thermal and mechanical processes. ${ }^{1}$ Initial attempts to create fistulas were"with an ab interno technique using argon lasers. ${ }^{2}$ This initial study in animal eyes revealed that while sclerostomies were successfully formed, collateral thermal damage was significant around the sclerostomy site and stimulated wound healing. The technique of flexible fibre optic probes with either quartz or sapphire tips, inserted across the anterior chamber to create a fistula in the opposite chamber angle, is also used in continuous wave YAG lasers to create similar fistulas. There has been initial work performed on animal eyes by Fankhauser et al. Clinical trials of this approach have been performed with some success. ${ }^{3}$ Wilson's series showed a continuing effect of the sclerostomy in 3 of 5 eyes 24 months after laser treatment in eyes with glaucoma and chronic inflammation. The technique does include entry and manipulation across the anterior chamber, which may cause cataract formation in phakic eyes as well as possible damage to collateral tissue by both mechanical and thermal means.

A gonioscopic delivery of laser energy is another approach, which has been investigated by Migdal, Latina and other workers. ${ }^{4,5}$ The use of a pulsed dye laser emitting red light at $590-660 \mathrm{~nm}$ is combined with iontophoresis staining of the fistula site sclera with methylene blue or a similar dye which absorbs the laser energy efficiently. The dyed sclera undergoes photoablation resulting in a tunnel sclerostomy. This method has the advantage of being the least invasive in terms of the laser energy introduction, but needs prior iontophoresis and is technically more difficult as visualisation must be maintained throughout the procedure and end point determination may be obscured by debris in the laser site. Visible sclerostomies were made in $82 \%$ of Migdal's series of 11 patients, while Latina achieved 55\% patent sclerostomies in a series of 35 eyes.

The ab externo technique of THC holmium laser sclerostomy has been well studied by Hoskins and Vassiliadis. ${ }^{6}$ This technique uses a fibre optic probe emitting laser energy at right angles. Inserted under the conjunctiva and tunnelled to the limbus it creates a fistula by emitting infrared radiation at $2100 \mathrm{~nm}$ as repeated pulses. The holmium produces less collateral heat damage to tissue and is absorbed readily by water-containing tissues, creating a fairly clean tunnel on histological examination. Hoskins' study reported successful sclerostomies in 13 of 21 eyes at 6 months after laser treatment. The ab externo approach has the advantage of no intraocular manipulation, but the tunnelling of the probe under the conjunctiva to reach the limbus creates unavoidable conjunctival manipulation. In eyes with conjunctival scarring from previous surgery, the probe would need to be inserted with caution to avoid conjunctival tears. The procedure is also complicated by iris incarceration in the sclerostomy site in some cases.

The bandwagon is joined by excimer laser partial external trabeculoplasty, in which the external sclera overlying the trabecular meshwork is removed by photoablation using ultraviolet laser radiation at $193 \mathrm{~nm}$ derived 


\section{NON-SURGICAL DRAINAGE PROCEDURES}

from argon fluoride gas. ${ }^{7}$ There is still the necessity of creating a fornix-based conjunctival flap to expose the surgical site, and at present it seems to be essentially a high-tech alternative way of creating a sclerostomy. ${ }^{8,9}$ However, as the excimer has the advantage of little collateral damage and very accurate tissue ablation, more clinical studies of its use would seem to be indicated. ${ }^{1}$

None of these techniques bypasses what remains the main cause of filtration surgery failure: subconjunctival fibrosis of the bleb site. It may be argued that less manipulation of the conjunctiva will incite less inflammatory response than conventional surgery, but the chronic inflammation present in cases of uveitic glaucoma or rubeotic glaucoma needing surgery will probably still necessitate the combination of antimetabolites with these surgical procedures for better results. ${ }^{9}$ It should also be borne in mind that most of the techniques are not designed to deal with patients with chronic angle closure glaucomas. This limitation in the adaptability of the methods is a drawback. In addition, there is the view that performance of a full-thickness unguarded sclerostomy is a retrogressive step. All of these methods will need more detailed clinical trials to evaluate fully the optimal sclerostomy size for maximising effectiveness and minimising complications. With time and increasing experience, a more balanced view of the abilities and limitations of these new non-surgical procedures will emerge, allowing ophthalmologists to determine better their place in the armamentarium of fistulising surgery.

PAUl Chew

\section{REFERENCES}

1. Allan BDS, Van Saarloos PP, Cooper RL, Constable IJ. Laser microsclerostomy for primary open angle glaucoma: a review of laser mechanisms and delivery systems. Eye 1992;6:257-66.

2. Jaffe GJ, Williams GA, Mieler WF, Radius RL. Ab interno sclerostomy with a high powered argon laser. Am J Ophthalmol 1988; 106:391-6.

3. Wilson RP, Javitt JC. Ab interno laser sclerostomy in aphakic patients with glaucoma and chronic inflammation. Am J Ophthalmol 1990;110:178-84.

4. de Vivero C, Ruben S, Migdal C. Ab interno pulsed dye laser sclerostomy for the treatment of glaucoma: preliminary results of a new technique [abstract]. In: Glaucoma Group of UK and Eire, 13th annual meeting, November 1992.

5. Latina MA, Melamed S, March WF, Kass MA, Kolker AE. Gonioscopic ab interno sclerostomy. Ophthalmology 1992;99:1736-44.

6. Hoskins HD, Iwach AG, Vassiliadis A, Drake MV, Hennings DR. Subconjunctival THC:YAG laser thermal sclerostomy. Ophthalmology 1991;98:1394-9.

7. Seiler T, Kriegerowski M, Patmore A, Marshall J. Partial external trabeculectomy with the excimer laser: an experimental investigation of new treatment for glaucoma. Laser Light Ophthalmol 1990;3:97-109.

8. Spiegal D. New aspects of laser treatment of glaucoma. Curr Opin Ophthalmol 1991;2:151-4.

9. Carenini BB, Nuzzi R. Laser treatment of glaucoma. Curr Opin Ophthalmol 1992;3;178-89. 